\title{
Fronto-limbic neural variability as a transdiagnostic correlate of emotion dysregulation
}

\author{
Valeria Kebets ${ }^{1,2,3}{ }^{凶}$, Pauline Favre ${ }^{4,5}$, Josselin Houenou ${ }^{4,5}$, Mircea Polosan ${ }^{6}$, Nader Perroud ${ }^{1}$, Jean-Michel Aubry ${ }^{1}$, \\ Dimitri Van De Ville (iD ${ }^{2,7}$ and Camille Piguet ${ }^{1,5}$
}

(c) The Author(s) 2021

Emotion dysregulation is central to the development and maintenance of psychopathology, and is common across many psychiatric disorders. Neurobiological models of emotion dysregulation involve the fronto-limbic brain network, including in particular the amygdala and prefrontal cortex (PFC). Neural variability has recently been suggested as an index of cognitive flexibility. We hypothesized that within-subject neural variability in the fronto-limbic network would be related to inter-individual variation in emotion dysregulation in the context of low affective control. In a multi-site cohort ( $N=166,93$ females) of healthy individuals and individuals with emotional dysregulation (attention deficit/hyperactivity disorder (ADHD), bipolar disorder (BD), and borderline personality disorder (BPD)), we applied partial least squares (PLS), a multivariate data-driven technique, to derive latent components yielding maximal covariance between blood-oxygen level-dependent (BOLD) signal variability at rest and emotion dysregulation, as expressed by affective lability, depression and mania scores. PLS revealed one significant latent component $(r=$ $0.62, p=0.044$ ), whereby greater emotion dysregulation was associated with increased neural variability in the amygdala, hippocampus, ventromedial, dorsomedial and dorsolateral PFC, insula and motor cortex, and decreased neural variability in occipital regions. This spatial pattern bears a striking resemblance to the fronto-limbic network, which is thought to subserve emotion regulation, and is impaired in individuals with ADHD, BD, and BPD. Our work supports emotion dysregulation as a transdiagnostic dimension with neurobiological underpinnings that transcend diagnostic boundaries, and adds evidence to neural variability being a relevant proxy of neural efficiency.

Translational Psychiatry (2021)11:545; https://doi.org/10.1038/s41398-021-01666-3

\section{INTRODUCTION}

Emotion regulation allows individuals to modulate, manage or organize emotions in order to help them meet the demands of the environment and achieve their goals [1-3], implicating various processes and systems (e.g., cognitive, behavioral, social, biological). In contrast, emotion dysregulation has been described as "a pattern of emotional experience and/or expression that interferes with appropriate goal-directed behavior" [4]. Emotion dysregulation is a central feature of psychopathology, and is key to both the development and maintenance of mood, personality and anxiety disorders, among others [5-7]. Because it is both a risk factor for psychopathology in the general population, and is common across many forms of psychiatric disorders, a better understanding of the neurobiological underpinnings of emotion dysregulation may have important clinical applications, for instance in predicting or measuring the efficacy of a therapeutic intervention $[8,9]$ in a transdiagnostic setting.

Emotion regulation is thought to rely on a fronto-limbic network, whereby the prefrontal cortex (PFC) exerts cognitive control over the amygdala, a subcortical structure that is central to emotion processing and salience perception [10-12]. Unsurprisingly, alterations in this network are central to the pathophysiology of bipolar disorder (BD), borderline personality disorder (BPD), and attention deficit/hyperactivity disorder (ADHD), which are all characterized by emotion dysregulation [13-18]. Notably, the three disorders also share risk factors, such as childhood trauma and genetic overlap [19-22]. This suggests that emotion dysregulation might have underlying neurobiological mechanisms that are shared across these disorders.

A measure that has received increasing attention in the past few years is neural variability, obtained by computing withinsubject BOLD signal variability over the timecourse. First considered as neural "noise", it has since been proposed as an index of local system dynamics [23]. Indeed, a certain level of instability is thought to be required for the brain to flexibly explore different functional network configurations and adapt to various environmental demands [24-26]. Neural variability has been shown to vary with age [27-32], task performance

\footnotetext{
${ }^{1}$ Department of Psychiatry, University of Geneva, Geneva, Switzerland. ${ }^{2}$ Department of Radiology and Medical Informatics, University of Geneva, Geneva, Switzerland. ${ }^{3}$ Department of Electrical and Computer Engineering, National University of Singapore, Singapore, Singapore. ${ }^{4}$ Laboratoire Neuro-psychiatrie, AP-HP, Département MédicoUniversitaire de Psychiatrie et d'Addictologie (DMU IMPACT), Hôpital Henri Mondor, Fondation FondaMental, Université Paris Est Créteil (UPEC), INSERM U955, IMRB, 94010 Créteil, France. ${ }^{5}$ Université Paris Saclay, CEA, UNIACT, Neurospin, 91191 Gif-sur-Yvette, France. ${ }^{6}$ Department of Psychiatry and Neurology, CHU Grenoble Alpes, Univ. Grenoble Alpes, Inserm, U1216, GIN, 38000 Grenoble, France. ${ }^{7}$ Institute of Bioengineering/Center for Neuroprosthetics, Ecole Polytechnique Fédérale de Lausanne (EPFL), Lausanne, Switzerland. ${ }^{\times}$email: valkebets@gmail.com
} 
[28, 30, 33, 34], but also symptom severity [35-38]. However, these relationships are often not linear (e.g., inverted U-shape in development and aging), and are task-, difficulty-, and circuitdependent [33, 39, 40]. Nevertheless, this body of work demonstrates the functional relevance of neural variability, and that it can provide meaningful information that is complementary to mean-based measures.

Studies investigating neural variability in clinical populations have implicated neural circuits that are relevant for psychopathology. Indeed, brain signal variability in the medial PFC during rest was shown to correlate positively with increased ADHD symptoms and inattention in children with and without ADHD [38]. Furthermore, brain signal variability has been shown to vary with mood shifts. In patients with BD, opposing patterns of neural variability were found in the default and sensorimotor networks (SMN) between patients in the depressed and manic phases [37]. This pattern mirrored the psychomotor behavior (i.e., acceleration/ slowing), as well as the affective state (i.e., external/internal focus) that characterize the manic and depressive phases, respectively. Similarly, higher brain signal variability in the SMN was shown in individuals with a cyclothymic temperament compared to those with a depressive temperament in the general population [35]. Interestingly, it was suggested that increased neural variability in specific circuits might facilitate local neuronal responses to incoming stimuli, and lead to over-excitation of specific behaviors/symptoms, e.g., psychomotor behavior, ruminations [35, 37]. However, to date, most studies looking at neural variability have relied on case-control comparisons, and few have tested for transnosographic, dimensional relationships.

In contrast to traditional case-control reports, a recent movement in psychiatry has advocated for a dimensional approach in the search for neurobiological markers of psychiatric symptoms. The NIMH's Research Domain Criteria (RDoC) framework is one of the initiatives working towards developing a neurobiologicallybased classification of mental disorders that integrates findings from behavioral science, neuroscience, and genetics [41, 42]. Consequently, we favored a transdiagnostic approach in the present work by leveraging a multi-site cohort of healthy individuals and individuals suffering from conditions strongly associated with emotion dysregulation (ADHD, BD, and BPD). We aimed to identify an emotion dysregulation dimension with associated patterns of blood-oxygen level-dependent (BOLD) variability, present in varying degrees among all individuals from our transdiagnostic cohort, which would suggest common neurobiological mechanisms that transcend diagnostic boundaries. We relied on partial least squares, a multivariate data-driven technique that extracts latent components by maximizing covariance between spatial patterns of neural variability and behavior (here, emotion dysregulation, as expressed by a combination of affective lability, depression and mania assessments). More specifically, we hypothesized that neural variability in the fronto-limbic circuit would be related to individual variation in emotion dysregulation.

\section{MATERIALS AND METHODS Participants}

Data for this study were collected from three sites (Geneva, Paris, Grenoble; see Fig. S1 for participants' inclusion and exclusion criteria). All participants gave their written informed consent. The research was conducted according to the principles of the Declaration of Helsinki and was approved by the University of Geneva research Ethics Committee (CER 13-081), the Paris CPP Ile de France IX Ethics Committee, and the Grenoble University Hospital Ethics Committee ( $\mathrm{n}^{\circ}$ 2011-A00425-36). Inclusion criteria for all participants were age between 18 and 55, no history of alcohol or drug abuse/dependence, no current or past cardiac or neurological disease. Exclusion criteria for all participants were a history of neurological disease or head trauma with loss of consciousness, any significant cerebral anatomic abnormality, and contraindications for MRI.
Individuals with BD were recruited from the outpatient Mood Disorder Program of the Geneva University Hospital, from two university-affiliated participating centers (AP-HP, Henri Mondor Hospitals Créteil and Fernand Widal-Lariboisière Hospitals, Paris, France), and from the expert center for BD of Grenoble University Hospital. The clinical diagnosis was established using the DSM-IV-TR criteria by specialized psychiatrists and confirmed by the Mini-International Neuropsychiatric Interview [43], the Structured Clinical Interview for the DSM-IV [44], or the Diagnostic Interview for Genetic Studies (DIGS) [45]. Individuals were under stable medication for four weeks. Patients in Grenoble and Geneva were included in the study if they reported having been euthymic for at least 1 month prior to scanning and if they had a MADRS score $<15$ and a YMRS score $<7$. Patients in Paris were not in the acute phase of $\mathrm{BD}$ at the time of scanning.

Individuals with BPD and ADHD were recruited from the outpatient Emotional Dysregulation Unit for BPD and ADHD of the Geneva University Hospital. BPD diagnosis was established with the SCID for DSM-IV Axis II Personality Disorders [46], and ADHD diagnosis with the Diagnostic Interview for ADHD in Adults (DIVA 2.0), by trained clinicians as part of the standard procedure of these specialized programs. Some patients were under psychotropic medication for comorbidities, as reported in Table 1. Participants were instructed not to take psychostimulants on the day of the study data acquisition.

Control participants were recruited via local databases as well as through web advertisement and were matched with patients in terms of age, sex, level of education, and handedness. Exclusion criteria were past or present neurological or psychiatric disorders (Geneva, Grenoble), personal or family history of Axis I mood disorder, schizophrenia, or schizoaffective disorder (Paris), use of psychotropic medication, and contraindication for MRI. All participants underwent clinical assessment by trained raters using the DIGS [45].

In total, 122 individuals with BD were recruited on all three sites, 93 healthy controls $(\mathrm{HC})$ were recruited on two sites (i.e., Geneva and Paris), while 24 individuals with BPD and 21 individuals with ADHD were only recruited on one site (i.e., Geneva). We excluded ten participants (8 BD, 1 $\mathrm{BPD}$, and $1 \mathrm{HC}$ ) for excessive in-scanner motion; 69 participants (39 BD, 2 $\mathrm{BPD}, 1 \mathrm{ADHD}$, and $27 \mathrm{HC}$ ) because they had not completed the clinical measures of interest; nine participants because they scored above 15 on the MADRS (7 BD, 2 BPD) and 6 because they scored above 7 on the YMRS (5 BD, $1 \mathrm{HC}$ ). The final sample thus comprised 166 participants, including 63 euthymic BD, 20 ADHD, 19 BPD, and 64 HC. The demographic, imaging, and clinical data of the final sample are shown in Table 1.

\section{Clinical assessment}

We used the Affective Lability Scales (ALS [47]), the Montgomery-Åsberg Depression Rating Scale (MADRS [48]), and the Young Mania Rating Scale (YMRS [49]) to measure different facets of emotion dysregulation. The ALS specifically measures affective lability, which refers to the frequency, speed, and range of changes in affective states [50]. The ALS is a 54-item self-reported questionnaire on which participants rate the tendency of their mood to shift between a "normal state" and different affects (depression, anger, anxiety, and elation), as well as their tendency to experience shifts between elation and depression, and between anxiety and depression. The total score was obtained by averaging across the six subscales, i.e., anger, anxiety, anxiety/depression, depression, depression/ elation, elation. The MADRS and YMRS are both clinician-rated scales that evaluate depressive and manic symptoms, respectively. The total score (sum across all items) was used for both scales.

\section{Magnetic resonance imaging acquisition}

Briefly, participants were scanned on 3T MRI scanners (see detailed MRI acquisition parameters in the Supplementary Methods). A resting state (RS) functional magnetic resonance imaging (fMRI) sequence, as well as an anatomical scan were acquired in all participants.

\section{Resting state fMRI preprocessing}

The first ten RS functional images were discarded to ensure signal equilibration, and the remaining images were preprocessed using SPM12 tools (http://fil.ion.ucl.ac.uk/spm/software/spm12). Functional images were first realigned, followed by co-registration of the mean functional image with the anatomical scan. Functional images were normalized to the MNI space with SPM12 "Segment", resampled to $3 \mathrm{~mm}$ isotropic voxels, and then spatially smoothed with a $6 \mathrm{~mm}$ full-width-at-half-maximum (FWHM) Gaussian filter. The average signal within a mask of white matter (WM) and cerebrospinal fluid (CSF) were extracted using the Data Processing 
Table 1. Demographic, imaging, and clinical profile of the sample used in the analyses $(N=166)$.

\begin{tabular}{|c|c|c|c|c|c|c|}
\hline & Bipolar $(N=75)$ & ADHD $(N=20)$ & Borderline $(N=21)$ & Controls $(N=65)$ & F/chi ${ }^{2}$ & $p$ val \\
\hline \multicolumn{7}{|l|}{ Demographics } \\
\hline Age, mean (SD) & $37.22(11.61)$ & $24.00(3.45)$ & $27.05(4.67)$ & $35.06(12.01)$ & 10.82 & $1.61 E-06$ \\
\hline $\operatorname{Sex}(F / M)$ & $30 / 33$ & $7 / 13$ & $19 / 0$ & $37 / 27$ & 20.39 & $1.41 E-04$ \\
\hline Education, mean (SD) ${ }^{\mathrm{a}}$ & $13.22(2.70)$ & $16.00(2.81)$ & $14.84(3.18)$ & $12.67(2.86)$ & 8.34 & 3.97E-05 \\
\hline \multicolumn{7}{|l|}{ Imaging } \\
\hline Scanner $(1 / 2 / 3 / 4)$ & $16 / 21 / 10 / 16$ & $20 / 0 / 0 / 0$ & $19 / 0 / 0 / 0$ & $16 / 48 / 0 / 0$ & 68.21 & $1.03 E-14$ \\
\hline \multicolumn{7}{|l|}{ Clinical } \\
\hline ALS, mean (SD) & $1.04(0.64)$ & $1.12(0.48)$ & $1.80(0.46)$ & $0.42(0.40)$ & 39.44 & $3.44 E-19$ \\
\hline MADRS, mean (SD) & $4.84(4.51)$ & $3.55(3.44)$ & $7.68(3.45)$ & $1.23(2.17)$ & 20.90 & $1.69 E-11$ \\
\hline YMRS, mean (SD) & $1.68(1.87)$ & $0.00(0.00)$ & $1.47(1.39)$ & $0.66(1.39)$ & 8.77 & $2.00 E-05$ \\
\hline \multicolumn{7}{|l|}{ Disease severity } \\
\hline Serotonergic, No. (\%) & 46 (73\%) & $1(5 \%)$ & $2(11 \%)$ & $0(0 \%)$ & - & - \\
\hline Glutamatergic, No. (\%) & $42(67 \%)$ & $0(0 \%)$ & $0(0 \%)$ & $0(0 \%)$ & - & - \\
\hline GABAergic, No. (\%) & $36(57 \%)$ & $0(0 \%)$ & $0(0 \%)$ & $0(0 \%)$ & - & - \\
\hline Norepinephrinergic, No. (\%) & $30(48 \%)$ & $15(75 \%)$ & $0(0 \%)$ & $0(0 \%)$ & - & - \\
\hline Lithium, No. (\%) & $38(60 \%)$ & $0(0 \%)$ & $0(0 \%)$ & $0(0 \%)$ & - & - \\
\hline No medication, No. (\%) & $30(48 \%)$ & $5(25 \%)$ & 17 (89\%) & $46(72 \%)$ & - & - \\
\hline Medication load, mean (SD) & $2.14(1.51)$ & $0.85(0.59)$ & $0.16(0.50)$ & $0.31(0.53)$ & - & - \\
\hline
\end{tabular}

Groups were compared with either ANOVAs (for continuous measures) or chi-squared tests (for categorical measures). All $p$-values that survived false discovery rate $(F D R)$ correction $(q<0.05)$ are indicated in bold. Disease severity and medication use are only shown for informative purposes, but were not compared between groups.

${ }^{\mathrm{a}}$ Based on 138 participants.

based on 82 patients.

'Based on 55 patients.

${ }^{\mathrm{d}}$ Medication was sorted by the neurotransmitter system(s) affected by the medication used by participants, based on the Neuroscience-based Nomenclature (NbN-2 [91, 92], http://nbn2r.com/). The list of medications and their categorization can be found in Table S5. Note that percentages may add up to more than $100 \%$ because some individuals take more than one medication.

Assistant for Resting-State fMRI toolbox [51]. The effects of WM, CSF, and six motion parameters were regressed out from the time-course, and a bandpass filter $(0.01-0.10 \mathrm{~Hz})$ was applied. Motion scrubbing [52] was applied to correct for motion artefacts; i.e., framewise displacement (FD) was calculated as the sum of the absolute values of the six realignment parameters, and scans with a FD higher than $0.5 \mathrm{~mm}$, as well as one scan before and two scans after, were excluded from the analysis. Participants with a time-course containing less than 4 min of scanning were excluded (8 $\mathrm{BD}, 1 \mathrm{BPD}$, and $1 \mathrm{HC}$ ).

\section{DARTEL group template}

A group template was generated with the Diffeomorphic Anatomical Registration Through Exponentiated Lie Algebra (DARTEL [53]) from the gray matter and white matter tissue segments of all the participants comprising the entire original sample ( $N=250$, see Fig. S1). Participants' T1 images were first segmented using the Computational Anatomy Toolbox (CAT12; http://www.neuro.uni-jena.de/cat/) "Segment Data" and the tissue segments were normalized to the tissue probability maps by means of an affine transformation. The group template was then normalized to the MNI space, and additional registration to the ICBM template was applied. Finally, the template was downsampled in order to match the dimensions of the functional images, and then binarized to include only voxels with a $\geq 50 \%$ gray matter probability.

Because of incomplete cerebellar coverage in 33 participants, we decided to exclude the cerebellum from the DARTEL template. To do so, we used a bilateral mask of the cerebellum as defined in Hammers atlas $[54,55]$, a probabilistic anatomical atlas based on 83 manually-delineated regions drawn on MR images of 30 healthy adult subjects. In order to encompass the whole cerebellum, we first smoothed the mask with a $25 \mathrm{~mm}$ FWHM Gaussian filter, then downsampled the mask to match the dimensions of the DARTEL template, and excluded the cerebellum mask from the DARTEL template.

\section{BOLD signal variability}

Voxel-wise BOLD signal variability was obtained for each participant by computing the standard deviation of each preprocessed time-course. This approach is equivalent to the frequency-domain computation of the amplitude of low-frequency fluctuations (ALFF) between 0.01 and $0.1 \mathrm{~Hz}$ $[56,57]$, and is strongly correlated with mean-square successive differences (MSSD) $[28,58]$. BOLD signal variability maps were constrained to the binarized DARTEL template (excluding the cerebellum), and were $z$-scored across all voxels included in the template within each participant [57]. Age, sex, scanner, and head motion (i.e., mean FD) were linearly regressed out from the imaging data prior to the PLS analysis using a general linear model on MATLAB.

\section{Partial least squares (PLS) analysis}

We used PLS analysis to identify BOLD signal variability spatial patterns related to emotion dysregulation across all participants. PLS is a multivariate data-driven statistical technique that aims to maximize covariance between two matrices $[59,60]$. The optimal relationship between the two data matrices is represented as latent components (LCs), which are weighted linear combinations of the original data that maximally covary with each other. A LC is characterized by a spatial pattern of neural variability and a behavioral pattern of affective lability, 
depression, and mania (imaging and behavioral saliences, respectively). By linearly projecting the imaging and behavioral measures of each participant onto their respective saliences, we obtain individual-specific brain and behavior scores, which reflect the participants' imaging and behavioral contribution to each LC. Importantly, the PLS analysis was agnostic on the diagnostic group, so that transdiagnostic brainbehavior associations could be extracted.

The statistical significance of the LCs was assessed by constructing a null distribution of the singular values using permutation testing (1000 permutations), whereby the behavioral data was permuted within each diagnostic group, so that latent components would not be driven by group differences. To determine which behavioral measures and voxels were driving the significant LC, we computed Pearson's correlations between the original imaging data and brain scores, as well as between the original behavioral measures and behavioral scores [61, 62]. A higher positive (or negative) correlation for a particular behavioral measure for a given LC indicates greater importance of the behavioral measure for the $L C$, while a higher positive (or negative) correlation for a particular imaging measure for a given LC indicates greater importance of that imaging value for the LC. We estimated confidence intervals for these correlations with a bootstrapping procedure that generated 1000 samples with replacement from participants' imaging and behavioral data, while accounting for diagnostic groups (i.e., bootstrap resampling was performed within each diagnostic group) in order to avoid spatial and behavioral patterns being driven by group differences, since our aim was to find transdiagnostic patterns of emotion dysregulation. Z-scores were computed by dividing each correlation coefficient by its bootstrap-estimated standard deviation, and were considered as strong contributors to LCs at absolute values $>3$, corresponding to a robustness at a confidence interval of approximately 99\% [60]. See Supplementary Methods for more details.

\section{Posthoc analyses}

Two-sample $t$-tests were performed to test whether brain and behavioral scores were different between participants from different diagnostic groups. Group differences in demographics, head motion, and clinical measures were tested using one-way analysis of variance (ANOVA; for continuous measures) or chi-squared tests (for categorical measures). We also tested if there were any significant associations between PLS brain (or behavioral) scores and disease severity, as well as medication use, using either Pearson's correlations (for continuous measures), or $t$-tests (for binary measures). All posthoc analyses were corrected for multiple comparisons at a false discovery rate (FDR) of $q<0.05$.

\section{Control analyses}

A number of control analyses were computed to assess the robustness of our results (detailed in the Supplementary Methods). Briefly, we used BOLD signal variability maps that included the cerebellum; we accounted for education level, early life trauma, or disease severity; we considered patients only; and we considered only participants from the one site that included individuals from all four diagnostic groups to control for scan effects. Finally, group differences and the impact of disease severity on the brain-behavior associations were examined.

\section{RESULTS}

\section{Neural variability correlates of emotion dysregulation}

We applied PLS to whole-brain BOLD signal variability and a combination of clinical measures that characterize emotion dysregulation in 166 participants that were either healthy or had a diagnosis of ADHD, BD, or BPD. PLS revealed one significant latent component (LC1). LC1 revealed a significant association $(r=0.62, p=0.044)$ between BOLD signal variability and emotion dysregulation (Fig. 1a), accounting for $74 \%$ of the covariance between the two matrices.

Healthy individuals had significantly lower brain and behavior scores compared to all patient groups (Fig. 1b). Moreover, individuals with BPD had significantly higher brain and behavior scores compared to individuals with $A D H D$ and $B D$. This dimension was therefore expressed more strongly by individuals with BPD, and less strongly by individuals with no psychiatric diagnosis, although there was a large overlap among individuals from all diagnostic groups (as shown on Fig. 1a).
Higher loadings on LC1 were associated with greater affective lability and depression, whereas mania did not yield a strong contribution (Fig. 1c and Table S1). On the imaging side, LC1 was characterized by increased brain signal variability in the left ventromedial PFC, bilateral dorsomedial PFC, subgenual ACC, bilateral amygdala, right hippocampus, bilateral motor cortex, and right insula, as well as decreased variability in occipital regions (Fig. 1d and Table S2 for the list of all reliable peaks and their MNI coordinates). This pattern partly recapitulates the emotion regulation network, which is known to be dysfunctional in ADHD, $B D$, and BPD [13-18]. We note however that LC1 also featured brain regions whose role in emotion dysregulation is not clear (e.g., occipital regions).

Post-hoc associations between brain (or behavior scores) and disease severity, as well as medication use, can be found in Table 2 and Table S3. Using medication affecting the serotonergic system was associated with higher behavioral scores. When categorizing medication use by medication class, the use of stimulants was also associated with higher brain scores.

\section{Control analyses}

Control analyses whereby we i) used BOLD signal variability maps including the cerebellum; ii) accounted for education level or early life trauma; iii) considered patients only (i.e., excluded control participants); and iv) considered only participants from one site, all yielded saliences that were similar to the original brain and behavior saliences (see Table S4), all showing the reliability of our findings. Detailed results are reported in the Supplementary Results, Tables S5-S7, and Figs. S2-S4.

\section{DISCUSSION}

In this work, we aimed to identify spatial patterns of neural variability related to emotion dysregulation in a multi-site transdiagnostic cohort, using a multivariate data-driven approach. We found that emotion dysregulation was associated with a pattern of increased BOLD signal variability in the ventromedial PFC, dorsomedial and dorsolateral PFC, amygdala, hippocampus, insula and motor cortex, and decreased BOLD signal variability in occipital regions. Our findings are in line with emotion dysregulation being a dimensional construct that spans across individuals with affective disorders such as ADHD, BD, and BPD, rather than being specific to any of these disorders. Importantly, the spatial pattern of brain signal variability associated with this dimension bears a compelling resemblance to the fronto-limbic circuit that is thought to subserve emotion regulation, and is impaired in ADHD, $B D$, and $B P D$. Our findings therefore add evidence to brain signal variability being a relevant proxy of neural efficiency, and support emotion dysregulation as a transdiagnostic dimension with neurobiological underpinnings that transcend diagnostic boundaries.

The patterns of brain signal variability associated with greater emotion dysregulation were mainly located in the fronto-limbic system, which plays a key role in emotional control/regulation $[12,63]$. Critically, abnormalities in the fronto-limbic network are thought to underpin emotion dysregulation in pathophysiological models of BD and BPD [13-16, 18]. The suggested mechanism involves hyper-activation of limbic regions responsible for emotion generation-in particular, the amygdala, hippocampus, and ventral striatum-, coupled with hypo-activation of the PFC, which is responsible for cognitive control. This circuit has shown structural abnormalities in individuals with BD [14, 64-66], BPD $[15,16]$, but also ADHD [67], e.g., altered volumes of the amygdala and hippocampus, and cortical thinning of the PFC. In BPD patients, abnormal patterns of activity in the amygdala, hippocampus, ventrolateral PFC, and dorsolateral PFC were shown during emotion processing $[15,16,68]$, but also at rest, where the ACC, medial PFC and dorsolateral PFC were found to be hyper- 


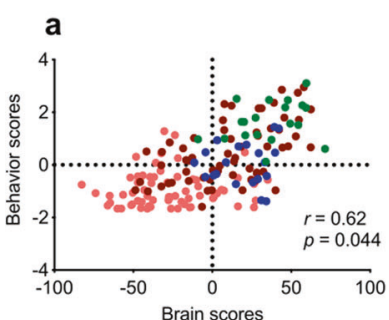

b - Controls : Bipolar

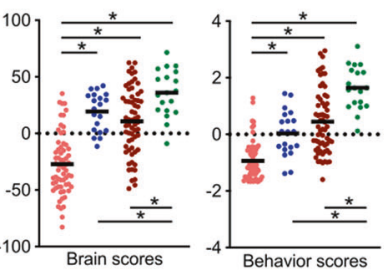

C

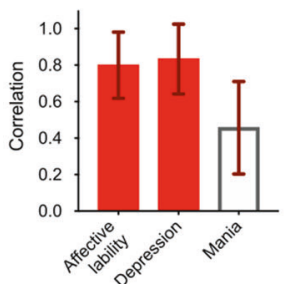

d

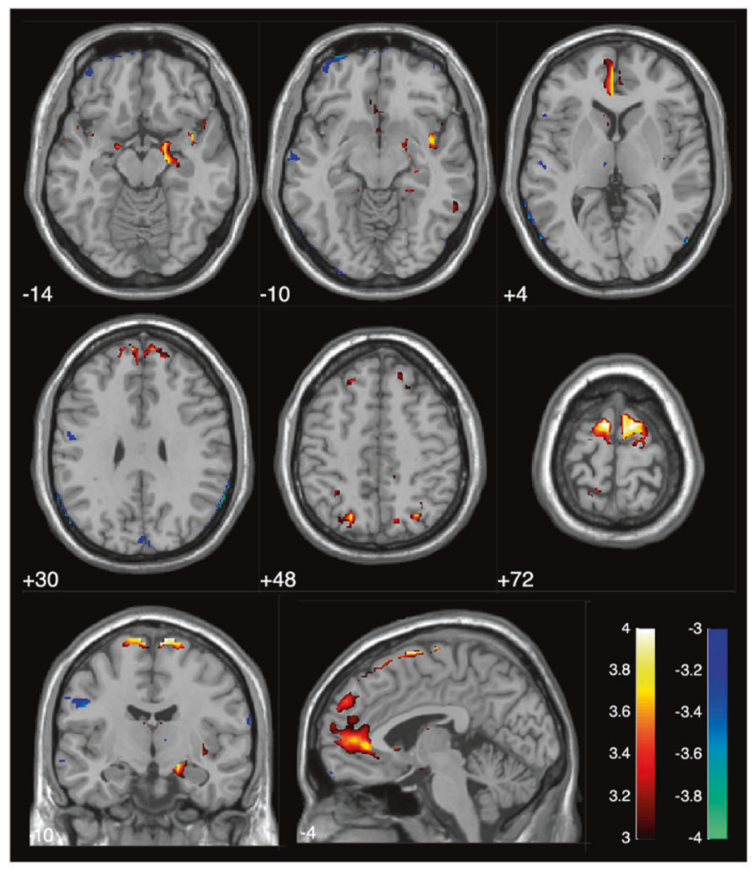

Fig. 1 The first latent component (LC1) is characterized by high emotion dysregulation. a PLS correlation between individual brain and behavior scores for LC1. Each dot is a participant from any of the four diagnostic groups. $\mathbf{b}$ Group differences in brain and behavior scores for LC1. Bold lines are mean scores for each group, while asterisks indicate two-sample $t$-tests that have survived to FDR correction ( $q<0.05$ ). Controls have significantly lower brain and behavior scores compared to all patient groups. BPD patients have significantly higher brain and behavior scores compared to ADHD and BD patients. $c$ Greater depression and affective lability characterize the behavioral pattern of LC1 (see Table S1). Mania did not have a strong contribution to LC1 $(z<3)$. Loadings are Pearson's correlations between participants' original behavioral data and their behavior scores, and error bars indicate bootstrap-estimated standard deviations. $\mathbf{d}$ LC1 is characterized by increased BOLD signal variability in the left ventromedial PFC, bilateral dorsomedial PFC, subgenual ACC, bilateral amygdala, right hippocampus, right insula, and bilateral motor cortex, as well as decreased BOLD signal variability in occipital regions (see Table S2 for MNI coordinates of peaks of all significant clusters). Loadings are $z$-scores obtained from bootstrapping, thresholded at absolute values $\geq 3(p<0.01)$.

activated during resting state in BPD patients compared to control participants [69]. Furthermore, neural activation and connectivity of fronto-limbic regions, especially the ACC, amygdala, insula, and ventrolateral PFC, showed changes following psychotherapy aimed at improving emotion regulation in BPD patients [70]. Abnormal patterns of activity and connectivity of fronto-limbic regions have been reported in euthymic $B D$ patients, especially involving the amygdala and the medial PFC at rest [71, 72], and during emotion regulation tasks $[73,74]$. Moreover, psychosocial intervention in individuals with $\mathrm{BD}$ or at risk for $\mathrm{BD}$ may induce functional and structural changes in these regions [75-77]. Emotion dysregulation is also prevalent in ADHD, and frontolimbic alterations involving the amygdala, orbitofrontal cortex, ventral striatum, and PFC have also been reported in this population [17]. We note however, that in addition to frontolimbic regions, spatial patterns of neural variability maps associated with LC1 also featured brain regions whose role in emotion dysregulation is not clear (e.g., occipital regions; see Table S3 for a list of the BOLD signal variability clusters that reliably contributed to $\mathrm{LC} 1$ ).

Previous studies of neural variability in $\operatorname{ADHD}[38,78-80], \mathrm{BD}$ $[37,81-86]$, and BPD patients $[87,88]$ have failed to show any consistent pattern, although some have reported alterations in regions of the fronto-limbic network (mostly the PFC). In ADHD patients, increased BOLD signal variability in the dorsolateral PFC, inferior frontal and orbitofrontal cortex were found during a Stroop task [78], as well as increased brain signal variability in the ventromedial PFC during a vigilance task in adolescents with ADHD compared to controls [80]. Moreover, greater MSSD in the dorsomedial PFC during rest was related to greater ADHD symptom severity, while greater MSSD in the ventromedial PFC was positively correlated with inattention across children with ADHD and typically developing children [38]. In BPD patients, increased ALFF was shown in the hippocampus [88], while increased ALFF in the ventral PFC, dorsolateral PFC, and insula were found in euthymic BD patients [85], compared to controls. The fronto-limbic circuit also overlaps with the DMN, in particular the ventromedial PFC and hippocampus. We found increased neural variability of the ventromedial PFC to be associated with LC1, which was mostly driven by greater levels of depression. This partly corroborates a previous study contrasting neural variability patterns in the DMN and SMN, i.e., higher DMN/SMN ratio in the depressive phase of $\mathrm{BD}$ and the inverse pattern during mania, which were positively correlated with depression and mania scores, respectively [37]. Therefore, our findings somewhat corroborate previous reports of altered neural variability in these clinical populations, but for the first time in a network directly associated with emotion regulation.

Our findings may be in apparent contrast to the prevailing view that brain signal variability facilitates neural flexibility by allowing fluid transitions between brain states via a stochastic resonance effect $[24,25]$. However, while it appears to be beneficial to task performance, heightened neural variability was also shown to correlate with worse clinical symptoms in various conditions [36-38]. As neural variability is thought to increase sensitivity to incoming stimuli, it is possible that heightened neural variability might lead to over-reactivity of specific neural circuits, as a maladaptive strategy to prepare for potentially relevant events, 
Table 2. Post-hoc associations between participants' brain (or behavioral) scores, disease severity, and medication use (categorized by the neurotransmitter target).

\begin{tabular}{|lrrrr|} 
& \multicolumn{2}{l}{ Brain scores } & \multicolumn{2}{c}{ Behavior scores } \\
& \multicolumn{1}{c}{$\boldsymbol{r} / \boldsymbol{t}$} & $\boldsymbol{p}$ & \multicolumn{1}{c}{$\boldsymbol{r} / \mathbf{t}$} & $\boldsymbol{p}$ \\
\hline Disease severity & & & & \\
\hline $\begin{array}{l}\text { Disease duration } \\
\text { \# Hospitalizations }\end{array}$ & 0.11 & 0.334 & 0.01 & 0.958 \\
\hline Medication use (by target) & -0.16 & 0.253 & 0.16 & 0.244 \\
\hline Dopaminergic & & & & \\
\hline Serotonergic & 2.40 & 0.018 & 2.10 & 0.037 \\
\hline Glutamatergic & -0.10 & 0.924 & 2.61 & 0.010 \\
\hline GABAergic & -0.18 & 0.860 & -0.04 & 0.969 \\
\hline Norepinephrinergic & -0.81 & 0.417 & 1.46 & 0.146 \\
\hline Lithium & 2.25 & 0.026 & 0.89 & 0.376 \\
\hline No medication & -1.87 & 0.064 & 0.73 & 0.467 \\
\hline \# Meds & 0.99 & 0.325 & -0.74 & 0.458 \\
\hline
\end{tabular}

Pearson correlations (for continuous measures) or t-tests (for categorical measures) were computed across all participants. A higher $r$ value indicates a stronger association between brain (or behavioral) scores and disease severity, while a higher $t$ value indicates higher brain (or behavioral) scores in participants taking the specific medication. Significant correlations or $t$-tests that survived FDR correction $(q>0.05)$ are indicated in bold. The same analysis was performed after classifying medication by medication class (see Table S3).

which instead supports the maintenance of emotion dysregulation in affective disorders. Consequently, our findings support the use of neural variability as a relevant proxy for dysfunctional emotional processing, which might be useful in tracking symptom severity and treatment efficacy [89].

The present study has several strengths including the use of a multivariate data-driven approach and the relatively large transdiagnostic cohort. Moreover, our approach aligns with recent initiatives such as the $\mathrm{RDoC}[41,42]$, that promote neurobiologically-based approaches to investigate dimensions of (ab)normal functioning, which often transcend classical nosological categories. Computational techniques may help in this endeavor by deriving brain-behavior associations that could serve as potential phenotypes. Moreover, by quantifying their neurobiological correlates, data-driven machine learning approaches such as PLS could help refine future taxonomies for mental disorders.

However, this work also has several limitations. First, because of poor cerebellar coverage in a number of participants, we decided to exclude the cerebellum from our analyses, even though an increasing number of reports implicate this structure in higherorder processes. Moreover, the diagnostic groups were not balanced across the three sites, which is a common shortcoming in multi-site studies with different protocols. Most patients were also using psychotropic medication at the time of scanning, which is known to modulate intrinsic brain activity metrics [90]. Our posthoc analyses showed mild associations $(t<3)$ between LC1 and medication use. Some control analyses were only exploratory, as sample sizes were small because of missing data (e.g., $N=55$ and $N=82$ when accounting for hospitalizations and disease duration, respectively). While PLS is a powerful technique for identifying brain-behavior associations, they remain correlational and do not claim to imply any causal relationship. Disease severity effects may have impacted our findings, in particular the number of hospitalizations which yielded high loadings when added to the clinical variables (see Supplementary Results), however we note that our cohort was not extensively phenotyped to investigate this question in depth, as many patients were missing this information (e.g., $N=55$ when accounting for hospitalizations). Finally, some of our control analyses suggest that LC1 might be partly driven by group differences between the patient groups and control participants. Future studies are needed to replicate and further investigate these effects.

Despite some limitations, our findings have unveiled the neural variability correlates of emotion dysregulation in the fronto-limbic system, further improving our understanding of the pathogenesis of affective disorders. Importantly, emotion dysregulation is a transdiagnostic construct that has shown clinical utility as a therapeutic target, as demonstrated by a decrease in maladaptive emotion regulation strategy use and symptom severity (e.g., depression, anxiety, substance use, etc.), regardless of the treatment protocol, the construct of emotion regulation that was examined, and the targeted disorder [9]. Indeed, transdiagnostic protocols aimed at improving emotion regulation have been shown to provide rapid and significant improvement in individuals with various forms of severe mental illness [8]. In this context, our approach might also provide a robust way of tracking therapeutic effects of interventions aimed at enhancing emotion regulation.

\section{CODE AVAILABILITY}

The code for the MRI preprocessing, BOLD signal variability extraction, as well as the PLS outputs can be found on https://github.com/valkebets/BOLDsd_ED, while the code for the PLS analysis is publicly available at https://github.com/danizoeller/ myPLS.

\section{REFERENCES}

1. Campos JJ, Campos RG, Barrett KC. Emergent themes in the study of emotional development and emotion regulation. Dev Psychol. 1989;25:394-402.

2. Gross JJ. The emerging field of emotion regulation: an integrative review. Rev Gen Psychol. 1998;2:271-99.

3. Hilt LM, Hanson JL, Pollack SD. Encyclopedia of adolescence. Amsterdam: Elsevier; 2011. p. $160-9$.

4. Beauchaine TP. Future directions in emotion dysregulation and youth psychopathology. J Clin Child Adolesc Psychol. 2015;44:875-96.

5. Aldao A, Nolen-Hoeksema S, Schweizer S. Emotion-regulation strategies across psychopathology: a meta-analytic review. Clin Psychol Rev. 2010;30:217-37.

6. Sheppes G, Suri G, Gross JJ. Emotion regulation and psychopathology. Annu Rev Clin Psychol. 2015;11:379-405.

7. Weissman DG, et al. Difficulties with emotion regulation as a transdiagnostic mechanism linking child maltreatment with the emergence of psychopathology. Dev Psychopathol. 2019;31:899-915.

8. Fowler JC, et al. Emotion dysregulation as a cross-cutting target for inpatient psychiatric intervention. J Affect Disord. 2016;206:224-31.

9. Sloan $\mathrm{E}$, et al. Emotion regulation as a transdiagnostic treatment construct across anxiety, depression, substance, eating and borderline personality disorders: a systematic review. Clin Psychol Rev. 2017;57:141-63.

10. Adolphs R. Neural systems for recognizing emotion. Curr Opin Neurobiol. 2002;12:169-77.

11. LeDoux JE. The emotional brain: the mysterious underpinnings of emotional life. New York: Simon and Schuster; 1996.

12. Ochsner KN, Silvers JA, Buhle JT. Functional imaging studies of emotion regulation: a synthetic review and evolving model of the cognitive control of emotion. Ann N Y Acad Sci. 2012;1251:E1-E24.

13. Chase HW, Phillips ML. Elucidating neural network functional connectivity abnormalities in bipolar disorder: toward a harmonized methodological approach. Biol Psychiatry Cogn Neurosci Neuroimaging. 2016;1:288-98.

14. Phillips ML, Swartz HA. A critical appraisal of neuroimaging studies of bipolar disorder: toward a new conceptualization of underlying neural circuitry and a road map for future research. Am J Psychiatry. 2014;171:829-43.

15. Ruocco AC, Carcone D. A neurobiological model of borderline personality disorder: systematic and integrative review. Harv Rev Psychiatry. 2016;24:311-29.

16. Schulze L, Schmahl C, Niedtfeld I. Neural correlates of disturbed emotion processing in borderline personality disorder: a multimodal meta-analysis. Biol Psychiatry. 2016;79:97-106.

17. Shaw $P$, Stringaris A, Nigg J, Leibenluft E. Emotion dysregulation in attention deficit hyperactivity disorder. Am J Psychiatry. 2014;171:276-93. 
18. van Zutphen L, Siep N, Jacob GA, Goebel R, Arntz A. Emotional sensitivity, emotion regulation and impulsivity in borderline personality disorder: a critical review of fMRI studies. Neurosci Biobehav Rev. 2015;51:64-76.

19. Moukhtarian TR, Mintah RS, Moran P, Asherson P. Emotion dysregulation in attention-deficit/hyperactivity disorder and borderline personality disorder. Borderline Personal Disord Emot Dysregulation. 2018;5:9.

20. Perroud $\mathrm{N}$, et al. Comorbidity between attention deficit hyperactivity disorder (ADHD) and bipolar disorder in a specialized mood disorders outpatient clinic. J Affect Disord. 2014;168:161-6.

21. van Hulzen KJE, et al. Genetic overlap between attention-deficit/hyperactivity disorder and bipolar disorder: evidence from genome-wide association study meta-analysis. Biol Psychiatry. 2017;82:634-41.

22. Witt $\mathrm{SH}$, et al. Genome-wide association study of borderline personality disorder reveals genetic overlap with bipolar disorder, major depression and schizophrenia. Transl Psychiatry. 2017;7:e1155-e1155.

23. Mclntosh AR, et al. The development of a noisy brain. Arch Ital Biol. 2010;148:323-37.

24. Deco G, Jirsa V, McIntosh AR, Sporns O, Kötter R. Key role of coupling, delay, and noise in resting brain fluctuations. Proc Natl Acad Sci. 2009;106:10302-7.

25. Ghosh A, Rho Y, Mclntosh AR, Kötter R, Jirsa VK. Noise during rest enables the exploration of the brain's dynamic repertoire. PLoS Comput Biol. 2008;4: e1000196.

26. McIntosh AR, Kovacevic N, Itier RJ. Increased brain signal variability accompanies lower behavioral variability in development. PLoS Comput Biol. 2008;4:e1000106.

27. Andrews-Hanna JR, et al. Disruption of large-scale brain systems in advanced aging. Neuron. 2007;56:924-35.

28. Garrett DD, Kovacevic N, McIntosh AR, Grady CL. The importance of being variable. J Neurosci. 2011;31:4496-503.

29. Garrett DD, Kovacevic N, Mclntosh AR, Grady CL. Blood oxygen level-dependent signal variability is more than just noise. J Neurosci 2010;30:4914-21.

30. Guitart-Masip $M$, et al. BOLD variability is related to dopaminergic neurotransmission and cognitive aging. Cereb Cortex. 2016;26:2074-83.

31. Nomi JS, Bolt TS, Ezie CEC, Uddin LQ, Heller AS. Moment-to-moment BOLD signal variability reflects regional changes in neural flexibility across the lifespan. J Neurosci. 2017;37:5539-48.

32. Samanez-Larkin GR, Kuhnen CM, Yoo DJ, Knutson B. Variability in nucleus accumbens activity mediates age-related suboptimal financial risk taking. J Neurosci. 2010;30:1426-34.

33. Armbruster-Genç DJN, Ueltzhöffer K, Fiebach CJ. Brain signal variability differentially affects cognitive flexibility and cognitive stability. J Neurosci. 2016;36:3978-87.

34. Raja Beharelle A, Kovačević N, Mclntosh AR, Levine B. Brain signal variability relates to stability of behavior after recovery from diffuse brain injury. NeuroImage. 2012;60:1528-37.

35. Conio $\mathrm{B}$, et al. Opposing patterns of neuronal variability in the sensorimotor network mediate cyclothymic and depressive temperaments. Hum Brain Mapp. 2019;40:1344-52.

36. Easson AK, McIntosh AR. BOLD signal variability and complexity in children and adolescents with and without autism spectrum disorder. Dev Cogn Neurosci. 2019;36:100630.

37. Martino $M$, et al. Contrasting variability patterns in the default mode and sensorimotor networks balance in bipolar depression and mania. Proc Natl Acad Sci. 2016;113:4824-9.

38. Nomi JS, et al. Resting-state brain signal variability in prefrontal cortex is associated with ADHD symptom severity in children. Front Hum Neurosci. 2018;12:90

39. Garrett DD, McIntosh AR, Grady CL. Brain signal variability is parametrically modifiable. Cereb Cortex. 2014;24:2931-40.

40. Mišić B, Mills T, Taylor MJ, Mclntosh AR. Brain noise is task dependent and region specific. J Neurophysiol. 2010;104:2667-76.

41. Cuthbert BN. The RDoC framework: facilitating transition from ICD/DSM to dimensional approaches that integrate neuroscience and psychopathology. World Psychiatry. 2014;13:28-35.

42. Insel T, Cuthbert B, Garvey M, Heinssen R, Pine DS, Quinn K, et al. Research domain criteria $(\mathrm{RDoC})$ : toward a new classification framework for research on mental disorders. Am J Psychiatry. 2010;167:748-51.

43. Sheehan DV, Lecrubier $Y$, Sheehan KH, Amorim $P$, Janavs J, Weiller $E$, et al. The Mini-International Neuropsychiatric Interview (M.I.N.I.): the development and validation of a structured diagnostic psychiatric interview for DSM-IV and ICD-10. J Clin Psychiatry. 1998;59:22-33.

44. First MB, Spitzer RL, Gibbon M, Williams JBW. Structured clinical interview for DSM-IV-TR axis I disorders, research version, patient edition (SCID-I/P). New York: Biometrics Research, New York State Psychiatric Institute; 2002.

45. Nurnberger $\mathrm{Jl}$, et al. Diagnostic interview for genetic studies. Rationale, unique features, and training. NIMH Genetics Initiative. Arch Gen Psychiatry. 1994;51:849-59.
46. First MB, Gibbon M, Spitzer RL, Benjamin LS. User's guide for the structured clinical interview for DSM-IV axis II personality disorders: SCID-II. Washington: American Psychiatric Press; 1997.

47. Harvey PD, Greenberg BR, Serper MR. The affective lability scales: development, reliability, and validity. J Clin Psychol 1989;45:786-93.

48. Montgomery SA, Åsberg M. A new depression scale designed to be sensitive to change. Br J Psychiatry. 1979;134:382-9.

49. Young RC, Biggs JT, Ziegler VE, Meyer DA. A rating scale for mania: reliability, validity and sensitivity. Br J Psychiatry J Ment Sci. 1978;133:429-35.

50. Aas M, et al. Psychometric properties of the Affective Lability Scale (54 and 18item version) in patients with bipolar disorder, first-degree relatives, and healthy controls. J Affect Disord 2015;172:375-80.

51. Yan C, Zang Y. DPARSF: a MATLAB toolbox for 'pipeline' data analysis of restingstate fMRI. Front Syst Neurosci. 2010;4:13.

52. Power JD, Barnes KA, Snyder AZ, Schlaggar BL, Petersen SE. Spurious but systematic correlations in functional connectivity MRI networks arise from subject motion. Neurolmage. 2012;59:2142-54.

53. Ashburner J. A fast diffeomorphic image registration algorithm. Neurolmage. 2007;38:95-113.

54. Gousias IS, Rueckert D, Heckemann RA, Dyet LE, Boardman JP, Edwards AD, et al Automatic segmentation of brain MRIs of 2-year-olds into 83 regions of interest. Neurolmage. 2008:40:672-84.

55. Hammers A, Allom R, Koepp MJ, Free SL, Myers R, Lemieux L, et al. Threedimensional maximum probability atlas of the human brain, with particular reference to the temporal lobe. Hum Brain Mapp. 2003;19:224-47.

56. Garrett DD, Samanez-Larkin GR, MacDonald SW, Lindenberger U, McIntosh AR, Grady $\mathrm{CL}$, et al. Moment-to-moment brain signal variability: a next frontier in human brain mapping? Neurosci Biobehav Rev. 2013;37:610-24.

57. Zöller D, Schaer M, Scariati E, Padula MC, Eliez S, Van De Ville D, et al. Disentangling resting-state BOLD variability and PCC functional connectivity in $22 \mathrm{q} 11.2$ deletion syndrome. Neurolmage. 2017;149:85-97.

58. Kielar A, Deschamps T, Chu RK, Jokel R, Khatamian YB, Chen JJ, et al. Identifying dysfunctional cortex: dissociable effects of stroke and aging on resting state dynamics in MEG and fMRI. Front Aging Neurosci. 2016;8:40.

59. Krishnan A, Williams LJ, Mclntosh AR, Abdi H. Partial least squares (PLS) methods for neuroimaging: a tutorial and review. Neurolmage. 2011;56:455-75.

60. McIntosh AR, Lobaugh NJ. Partial least squares analysis of neuroimaging data: applications and advances. Neurolmage. 2004;23:S250-263.

61. Courville T, Thompson B. Use of structure coefficients in published multiple regression articles: $\beta$ is not enough. Educ Psychol Meas. 2001;61:229-48.

62. Henson RK. The logic and interpretation of structure coefficients in multivariate general linear model analyses. 2002.

63. Ochsner KN, Gross JJ. The cognitive control of emotion. Trends Cogn Sci 2005;9:242-9.

64. Hanford LC, Nazarov A, Hall GB, Sassi RB. Cortical thickness in bipolar disorder: a systematic review. Bipolar Disord. 2016;18:4-18.

65. Hibar DP, et al. Cortical abnormalities in bipolar disorder: an MRI analysis of 6503 individuals from the ENIGMA Bipolar Disorder Working Group. Mol Psychiatry. 2018;23:932-42.

66. Hibar DP, et al. Subcortical volumetric abnormalities in bipolar disorder. Mol Psychiatry. 2016;21:1710-6.

67. Hoogman $M$, et al. Subcortical brain volume differences in participants with attention deficit hyperactivity disorder in children and adults: a cross-sectional mega-analysis. Lancet Psychiatry. 2017;4:310-9.

68. Ruocco AC, Amirthavasagam S, Choi-Kain LW, McMain SF. Neural correlates of negative emotionality in borderline personality disorder: an activation-likelihoodestimation meta-analysis. Biol Psychiatry. 2013;73:153-60.

69. Visintin $E$, et al. Mapping the brain correlates of borderline personality disorder: a functional neuroimaging meta-analysis of resting state studies. J Affect Disord. 2016:204:262-9.

70. Marceau EM, Meuldijk D, Townsend ML, Solowij N, Grenyer BFS. Biomarker correlates of psychotherapy outcomes in borderline personality disorder: a systematic review. Neurosci Biobehav Rev. 2018;94:166-78.

71. Favre $P$, Baciu M, Pichat $C$, Bougerol T, Polosan M. fMRI evidence for abnormal resting-state functional connectivity in euthymic bipolar patients. J Affect Disord. 2014;165:182-9.

72. Rey G, et al. Resting-state functional connectivity of emotion regulation networks in euthymic and non-euthymic bipolar disorder patients. Eur Psychiatry. 2016;34:56-63.

73. Favre $\mathrm{P}$, Polosan M, Pichat C, Bougerol T, Baciu M. Cerebral correlates of abnormal emotion conflict processing in euthymic bipolar patients: a functional MRI study. PLoS ONE. 2015;10:e0134961.

74. Rey G, Desseilles M, Favre S, Dayer A, Piguet C, Aubry JM, et al. Modulation of brain response to emotional conflict as a function of current mood in bipolar 
disorder: preliminary findings from a follow-up state-based fMRI study. Psychiatry Res Neuroimaging. 2014;223:84-93.

75. Favre P, Houenou J, Baciu M, Pichat C, Poupon C, Bougerol T, et al. White matter plasticity induced by Psychoeducation in bipolar patients: a controlled diffusion tensor imaging study. Psychother Psychosom. 2016;85:58-60.

76. Favre $P$, Baciu $M$, Pichat $C$, De Pourtalès MA, Fredembach $B$, Garçon $S$, et al. Modulation of fronto-limbic activity by the psychoeducation in euthymic bipolar patients. A functional MRI study. Psychiatry Res Neuroimaging. 2013;214:285-95.

77. Garrett AS, Miklowitz DJ, Howe ME, Singh MK, Acquaye TK, Hawkey CG, et al. Changes in brain activation following psychotherapy for youth with mood dysregulation at familial risk for bipolar disorder. Prog Neuropsychopharmacol Biol Psychiatry. 2015;56:215-20.

78. Depue BE, Burgess GC, Willcutt EG, Bidwell LC, Ruzic L, Banich MT. Symptomcorrelated brain regions in young adults with combined-type ADHD: their organization, variability, and relation to behavioral performance. Psychiatry Res Neuroimaging. 2010;182:96-102.

79. Mowinckel AM, Alnæs D, Pedersen ML, Ziegler S, Fredriksen M, Kaufmann T, et al. Increased default-mode variability is related to reduced task-performance and is evident in adults with ADHD. Neurolmage Clin. 2017;16:369-82.

80. Sørensen L, Eichele T, van Wageningen $\mathrm{H}$, Plessen $\mathrm{KJ}$, Stevens MC. Amplitude variability over trials in hemodynamic responses in adolescents with ADHD: the role of the anterior default mode network and the non-specific role of the striatum. Neurolmage Clin. 2016;12:397-404.

81. Liu C-H, Ma X, Wu X, Li F, Zhang Y, Zhou FC, et al. Resting-state abnormal baseline brain activity in unipolar and bipolar depression. Neurosci Lett. 2012;516:202-6.

82. Lu D, Jiao Q, Zhong Y, Gao W, Xiao Q, Liu X, et al. Altered baseline brain activity in children with bipolar disorder during mania state: a resting-state study. Neuropsychiatr Dis Treat. 2014;10:317-23.

83. Lui S, Yao L, Xiao Y, Keedy SK, Reilly JL, Keefe RS, et al. Resting-state brain function in schizophrenia and psychotic bipolar probands and their first-degree relatives. Psychol Med. 2015;45:97-108.

84. Meda SA, Wang Z, Ivleva El, Poudyal G, Keshavan MS, Tamminga CA, et al. Frequency-specific neural signatures of spontaneous low-frequency resting state fluctuations in psychosis: evidence from bipolar-schizophrenia network on intermediate phenotypes (B-SNIP) consortium. Schizophr Bull. 2015;41:1336-48.

85. Xu K, Liu H, Li H, Tang Y, Womer F, Jiang $X$, et al. Amplitude of low-frequency fluctuations in bipolar disorder: a resting state fMRI study. J Affect Disord. 2014;152-154:237-42.

86. Zhang Z, Bo Q, Li F, Zhao L, Wang Y, Liu R, et al. Increased ALFF and functional connectivity of the right striatum in bipolar disorder patients. Prog Neuropsychopharmacol Biol Psychiatry. 2020. https://doi.org/10.1016/j.pnpbp.2020.110140.

87. Lei $X$, Zhong $M$, Liu $Y$, Jin $X$, Zhou $Q, X i C$, et al. A resting-state fMRI study in borderline personality disorder combining amplitude of low frequency fluctuation, regional homogeneity and seed based functional connectivity. J Affect Disord. 2017;218:299-305.

88. Salvador R, Vega D, Pascual JC, Marco J, Canales-Rodríquez EJ, Aguilar S, et al. Converging medial frontal resting state and diffusion-based abnormalities in borderline personality disorder. Biol Psychiatry. 2016;79:107-16.

89. Dinstein I, Heeger DJ, Behrmann M. Neural variability: friend or foe? Trends Cogn Sci. 2015;19:322-8.

90. Pereira-Sanchez V, Franco AR, Vieira D, de Castro-Manglano P, Soutullo C, Milham $M P$, et al. Systematic review: medication effects on brain intrinsic functional connectivity in patients with attention-deficit/hyperactivity disorder. J Am Acad Child Adolesc Psychiatry. 2020. https://doi.org/10.1016/j.jaac.2020.10.013.

91. Zohar J, Stahl S, Moller HJ, Blier P, Kupfer D, Yamawaki S, et al. A review of the current nomenclature for psychotropic agents and an introduction to the neuroscience-based nomenclature. Eur Neuropsychopharmacol. 2015;25:2318-25.

92. Zohar J, Nutt DJ, Kupfer DJ, Moller HJ, Yamawaki S, Spedding M, et al. A proposal for an updated neuropsychopharmacological nomenclature. Eur Neuropsychopharmacol. 2014;24:1005-14.

\section{ACKNOWLEDGEMENTS}

We wish to thank Gwladys Rey for her initial help in gathering a multi-site database, Anne-Lise Küng and Eleonore Pham for their help in the recruitment of participants, and Alexandre Dayer for his inspirational guidance and enthusiasm for this project. He will stay in our hearts.

\section{AUTHOR CONTRIBUTIONS}

$\mathrm{CP}$ and JMA formulated the research question. VK, PF, JH, NP, JMA, DVDV, and CP conceptualized the project, and designed the study and methods. PF, JH, MP, NP, and $\mathrm{CP}$ collected the neuroimaging data, and performed the clinical assessment of participants. JMA provided institutional support. VK analyzed the data and wrote the manuscript. All the authors reviewed the manuscript and provided critical input.

\section{FUNDING}

This work was supported by the Swiss National Center of Competence in Research; "Synapsy: the Synaptic Basis of Mental Diseases", financed by the Swiss National Science Foundation (grant number 51NF40-158776), a grant of the Swiss National Science Foundation to JMA (grant number 32003B-156914) and the Fondamental Suisse Foundation. VK was partly supported by the Singapore National Research Foundation (NRF) Fellowship (Class of 2017) and the Singapore Ministry of Defense (Project CURATE). Her work utilized resources provided by the Center for Functional Neuroimaging Technologies, NIH P41EB015896 and instruments supported by NIH 1S10RR023401, NIH 1S10RR019307, and NIH 1S10RR023043 from the Athinoula A. Martinos Center for Biomedical Imaging at the Massachusetts General Hospital. The computational work was partially performed on resources of the National Supercomputing Centre, Singapore (https://www.nscc.sg). This work was supported by the French ANR under the "VIP" (MNP 2008) Project; the Investissements d'Avenir programs managed by the ANR under references ANR-11-IDEX-004-02 (Labex BioPsy) and ANR-10-COHO-10-01; and the Fondation pour la Recherche Médicale ("Bioinformatique pour la biologie 2014"). This work was also supported by research grants from Grenoble University Hospital (http://www.chu-grenoble.fr/). The Grenoble MRI facility IRMaGE was partly funded by the French program "Investissement d'avenir" run by the "Agence Nationale pour la Recherche" (http://www.agence-nationalerecherche.fr/): Grant "Infrastructure d'Avenir en Biologie Santé" (grant number ANR11-INBS-0006)

\section{COMPETING INTERESTS}

The authors declare no competing interests.

\section{ADDITIONAL INFORMATION}

Supplementary information The online version contains supplementary material available at https://doi.org/10.1038/s41398-021-01666-3.

Correspondence and requests for materials should be addressed to Valeria Kebets.

Reprints and permission information is available at http://www.nature.com/ reprints

Publisher's note Springer Nature remains neutral with regard to jurisdictional claims in published maps and institutional affiliations.

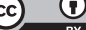

Open Access This article is licensed under a Creative Commons Attribution 4.0 International License, which permits use, sharing, adaptation, distribution and reproduction in any medium or format, as long as you give appropriate credit to the original author(s) and the source, provide a link to the Creative Commons license, and indicate if changes were made. The images or other third party material in this article are included in the article's Creative Commons license, unless indicated otherwise in a credit line to the material. If material is not included in the article's Creative Commons license and your intended use is not permitted by statutory regulation or exceeds the permitted use, you will need to obtain permission directly from the copyright holder. To view a copy of this license, visit http://creativecommons. org/licenses/by/4.0/.

(c) The Author(s) 2021 